The loop of the second traction sutare $B \quad b$ is now loosened from its clip, drawn tight, the ends tied and cut away. The first traction threads are cut, and releasing the vessel thereby from the frame, the suture is complete.

On loosening the temporary clamps, there is sometimes a little oozing at the suture line, which stops in a minute or two under gentle pressure with gauze between the fingers. The reason for laying and tying the sutures in a definite order is that if one breaks in the tying it cannot be replaced if the sutures on both sides of it have been already tied.

This method of end-to-end union of a divided vessel differs in some small but important details from that previously published by me.

The "frame" is a greater convenience as compared with the "measure" of the original method, in that the vessel once fixed is not removed till the suture is complete, the other side being exposed by simply sliding back the plate, and reversing the frame as described.

The main difference is that, in the original directions, the second suture inserted, which forms the traction threads $B b$ during the suturing of the two sides of the stretched vessel, is directed to be tied last of all, as in this method, but the ends of the chromic gat " quill" $\mathbf{G ~ G}^{1} j j^{1}$ were originally directed to be tied in this suture atter removal from the "measure" (which there takes the place of the "frame"), while now the two ends of the "quills" $\mathrm{G} \mathrm{G}^{1} j j^{1}$ are tied in the last of the sutures on the reverse side of the "frame," and so the diameter of the supporting s"quills," which determines that of the finished union, is fixed before the traction is released and the "frame" remored. The traction suture $\mathrm{B} b$ is then tied, the section of ressel wall concerned being already held stretched, whereas in the former method that section of wall was free to contract, the traction being released, and consequently the contraction led to a slight narrowing at the sature line.

1. The course of the suture in its passage through the walls of the apposed ends of the vessel is to be noted. If the details above described as to threading the needle and as to the distinction of the ends of the sutare before tying into upper $D$ (Fig. 5) and lower $d$, with respect to the strand of catgut $j$ over which they are tied, the suture will invariably take the form of the figure 8, the space in each

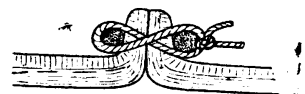

Fig. 7.-Shows diagrammatically the figure of 8 shape of the suture in situ.

half of the figare being occupied by the chromic catgat, while the cross in the centre is where the suture pierces the vessel walls (Fig. 7). There is thus no possibility of the suture cutting out on each side to the width of the thickness of the chromicized catgut, and the hole through which it passes in the vessel walls is minimal.

2. The catgut itself is a powerfal aid against any tendency of the suture to cat out, by giving a more extended hold on the vessel wall. It acts also as a pair of splints, keeping the two ondothelial surfaces in contact between the suture points.

3. Fewer sutures are required than under any other suture method, and so fewer needle punctures, and less consequent weakening of the wall of the vessel.

4. Nothing but an endothelial lining is exposed to the blood stream, even the sutures, though perforating all coats, being practically prevented from contact with blood.

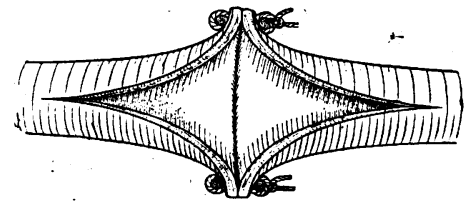

Fig. 8.-Diagrammatic representation of the internal appearance of the suture line in an artery which has been united by the method described.

The inner aspect of the vessel after suture is shown diagrammatically in Fig. 8.

5. If the observations of measurements of the size of the vessel when fall of blood, and when stretched in this "frame," are correctly made, there is a mathematical certainty of having the size of the vessel at the suture line that of ordinary distension under the pressure of the blood.

6. As I have shown in the book referred to, when bealing has occurred no suture material remains in $\varepsilon i t u$ in the vessel wall, the catgut being eventually absorbed, and the silk completely outside the vessel; in the scar itself new elastic and muscular fibres form, and there is a strong probability of a complete restitutio ad integrum being established.

I have repeatedly united by this method divided vessels of a diameter when distended with blood of not more than $3 \mathrm{~mm}$. Rarely can any necessity arise in human surgery for suture of a smaller vessel.

The method is equally applicable to end.to end suture of veins, though it is specially designed to furnish a eafe sutare under conditions of pressure such as exist in arteries.

A suture on the same principle is also applicable to the repair of wounds in the wall of a vessel not amounting to complete division. If the wound were longitudinal or slightly oblique the "frame" would not be required; if very oblique or transverse, it might be wiser to complete the division and make a union as above described.

It would then, of course, be necessary to observe the original diameter of the vessel under the arterial pressure by temporarily controlling its proximal end and laying it across the graduated plate of the "frame" when filled out with blood.

1 Suture of Arteries. By E. Archibald Smith. Oxford Medical Press. 1909.

\section{A NOTE UPON}

\section{THE MECHANICAL EFFECTS OF A MASSIVE RIGHT-SIDED PLEURAL EEFUSION.}

By A. C. GEDDES, M.D.

PROFESSOR OF ANATOMY AT THE ROYAL COLLEGE OF SURGEONS IN IRELAND.

(From the Anatomical Laboratory of the College)

THE observations here recorded were made on a dissecting room subject preserved by the double vascular injection method. The arteries were injected under low pressure with a corrosive-carbolic-glycerine preservative, the venae cavae and their tributaries to the first valves with 15 per cent. formalin.

This method of preservation secures that the majority of the tissues retain an almost lifelike consistency, whereas the heart, liver, and kidneys are firmly fixed and the lungs partially fixed.

The body upon which the observations were made was that of an old woman, whose age was returned as 67. Before dissection was commenced the right shoulder was seen to be considerably higher than the left. This eleration is shown in the photograph. When the thorax was opened, the right pleural cavity was found full of clear fluid. The condition of the (partially fixed) right lung is shown in the figure. It contained air, but was not completely expanded.

In connexion with the displacement of the thoracic viscera the most striking fact was that the inferior vena cava and the pericardial attachment of the diaphragm had moored the mediastinal structures as efficiently inferiorly to and through the diaphragm as the great vessels, trachea, and oesophagus moor them superiorly to the margins of the thoracic inlet. As a result the displace. ment of the mediastinal structures to the left was maximal at the level of the lung roots and minimal at the levels of the thoracic inlet and diaphragm.

The diaphragmatic pleural recesses were widely opened up, and the convexity of the right cupola of the diaphragm was in part reversed, so that it looked downward instead of upward. The posterior part of the mediastinal pleura was balged somewhat to the left of the middle line, bat not sufficiently to clear the vertebral bodies.

The heart was displaced, the apex having passed down. wards and slightly inwards. The mechanism of this displacement was: the right margin of the inferior or diaphragmatic surface of the heart was held almost im. 
movably by the inferior vena cava. As a result the pressure from the right was only able to displace to the left the upper part of the normal vertical axis of the heart, so that the apex was forced to descend. An adequate description of the cardiac displacement would be that the heart was rotated clockwise through an angle of 30 degrees on an anteroposterior axis which passed through the caval opening in the diaphragm. The photograph shows only a small part of this rotation, for the removal of the flaid withdrew the displacing force and the natural elasticity of the tissues effected a partial rectification. The full extent of the downward rotation of the apex can be accurately gauged from the inclination of the upper surface of the left lobe of the liver against which it was firmly pressed, the diaphragm alone intervening.

The margo acutus of the heart was deeply notched at about its middle. The causation of this notching is considered later in connexion with the distortion of the liver. The notch was deep enough seriously to affect the capacity of the right ventricle.

The right auricle was almost oblite rated, its cavity being represented by a mere slit. The superior vena cava was compressed and extended. Its lumen apparently was much reduced. The short ntrapericardial part of the inferior vens cava was compressed, extended, and sharply bent from right to left. It was difficult to understand how any appreciable quantity of blood could have entered the auricle. The left auricle was pulled out on its right side, and the right pulmonary veins were elongated.

As a whole, the heart was flattened on its right side. This flattening is shown in the photo. graph. The peri. cardium was healthy.

The vertical curv. ature of the aorta was of shorter radius than usual; this was clearly due to the rotation of the heart. The horizontal ourv ature was consider. ably flattened.

The liver was depressed but not rotated. The upper

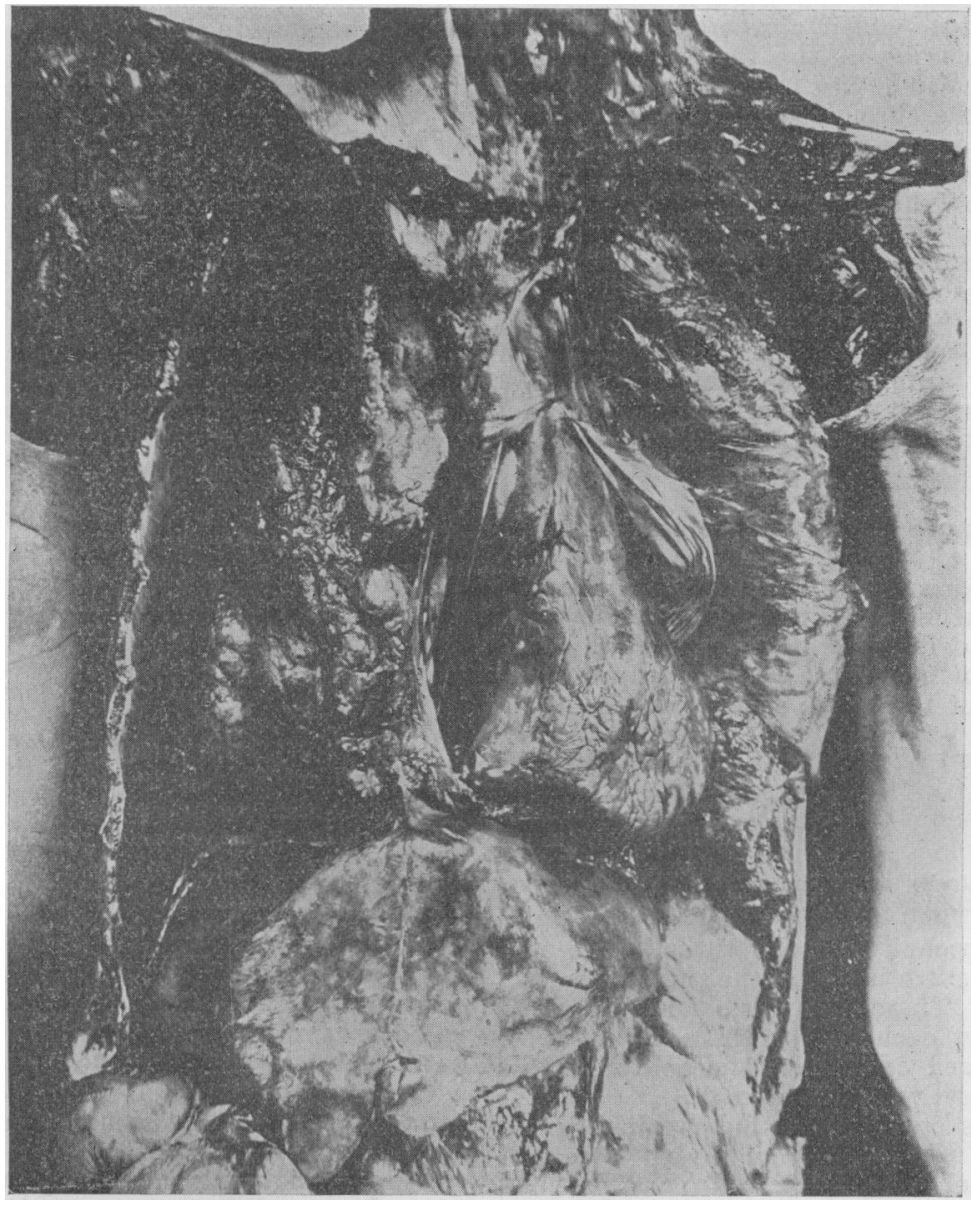
To show the flattening on the right side of the heart, the slope of the upper surface
the left lobe of the liver and the deep depression on the upper surface of the of the left lobe of the liver and the deep depression on the upper surface of the of the left as seriously affecting the capacity of the right venTo

methods of examination the downward displacement of the liver must have been in some measure masked, for the distended transverse colon lay in front of the lower third of the organ.

The descent of the liver had caused a considerable downward displacement of the right kidney. The descent of the diaphragm had carried the right suprarenal downwards. These displacements must of necessity have caused considerable traction upon the renal and suprarenal plexuses of the sympathetic nervous eystem. interesting briefly to compare the actual observations made in this case under the most favourable conditions upon hardened organs with some parts of a standard description of the mechanical effects of massive rightsided plearal effusion such as that given by Dr. West in his book, Diseases of the Organs of Respiration. He writes :

With effusion on the right side, the pleura extends across the sternum ... the line of dullness being tinued through the nipple nipple, or above and oulside it, to the spot where the apex of the

The photograph shows that in this case the line of dullness would have followed the line in. dicated, but that the dullness would not have been due to an extended right pleura contrining fluid, but to the displaced mediastinal structures. pushing forward and to the left behind the emptied anterior part of the left pleural cavity.

Thedescription con. tinues :

The most marked change on this (the right) side is in the position of the liver, which is greatly displaced and curiously rotated. The lower border reaches down as. far as the border of the ambilicus : * ; the notch is then usually close under the left costal arch and the gall bladder in the middle. line.

The downward displacement of the urface of its right lobe was deeply concave. This con. cavity was directly due to the transmission of the pressure of the pleural fluid through the diaphragm. The upper surface of the left lobe sloped steeply from a point near the middle line of the body downwards and to the left. This depression was due to the forced downward rotation of the apex of the heart. The central part of the liver held by its diaphragmatic attachment, the falciform liga. ment, and the hepatic veins, had resisted the general downward movement, if, indeed, it were not somewhat elevated. It appeared almost to have been squeezed upwards as a result of the action on the liver of three forces-the downward pressure of the fluid on the right, the downward pressure of the heart apex on the left, and the upward counter pressure of the intestines below. $\mathrm{Be}$ this as it may, the central part of the liver formed a prominent projecting rounded mass, against which the margo acutus was compressed. This was the canse of the notch which has already been described liver certainly occurred in this case, but there was no rotation. In view of the almost necessary descent of the apex of the heart, it is difficult to believe that. such rotation is the rule. The feeling of a notch near the left costal margin might be simulated by pal. pation of the depressed left lateral border of the organ.

In Dr. West's description the possibility of a rotatory of the right auricle and ventricle is somewhat lightly dismissed, and the possibility of kinking of the vena. cava inferior is categorically denied. It existed in this case, and certainly looked sufficiently effective to explain a sudden death-a possibility which was. originally suggested by Bartels. It is necessary, however, to remember in this connexion the compression of the right auricle and ventricle and the traction upon the renal and suprarenal sympathetic nervous plexuses. displacement of the heart is not discussed. Compression 